\title{
Ground-state and dynamical properties of two-dimensional dipolar Fermi liquids
}

\author{
Saeed H. Abedinpour ${ }^{\mathrm{a}, \mathrm{b}, *}$, Reza Asgari ${ }^{\mathrm{b}}$, B. Tanatar ${ }^{\mathrm{c}}$, \\ Marco Polini ${ }^{\mathrm{d}}$
}

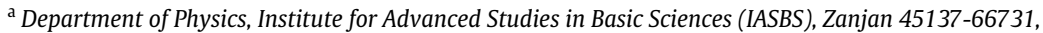

Iran

${ }^{\mathrm{b}}$ School of Physics, Institute for Research in Fundamental Sciences (IPM), Tehran 19395-5531, Iran

${ }^{\mathrm{c}}$ Department of Physics, Bilkent University, Bilkent, 06800 Ankara, Turkey

${ }^{\mathrm{d}}$ NEST, Istituto Nanoscienze-CNR and Scuola Normale Superiore, I-56126 Pisa, Italy

\section{H I G H L I G H T S}

- We have studied the ground state properties of a strongly correlated two-dimensional fluid of dipolar fermions.

- We have calculated the effective inter-particle interaction and the dynamical density-density response function.

- We have shown that an undamped zero sound mode exists at any value of the interaction strength.

\section{A R T I C L E I N F O}

\section{Article history:}

Received 29 March 2013

Accepted 16 October 2013

Available online 21 October 2013

\section{Keywords:}

Degenerate Fermi liquid

Ultracold atoms

Dipolar interaction

Zero-sound mode

\begin{abstract}
A B S T R A C T
We study the ground-state properties of a two-dimensional spinpolarized fluid of dipolar fermions within the Euler-Lagrange Fermi-hypernetted-chain approximation. Our method is based on the solution of a scattering Schrödinger equation for the "pair amplitude" $\sqrt{g(r)}$, where $g(r)$ is the pair distribution function. A key ingredient in our theory is the effective pair potential, which includes a bosonic term from Jastrow-Feenberg correlations and a fermionic contribution from kinetic energy and exchange, which is tailored to reproduce the Hartree-Fock limit at weak coupling. Very good agreement with recent results based on quantum Monte Carlo simulations is achieved over a wide range of coupling constants up to the liquid-to-crystal quantum phase transition. Using the fluctuation-dissipation theorem and a static approximation for the effective inter-particle interactions, we calculate the dynamical
\end{abstract}

\footnotetext{
* Corresponding author at: Department of Physics, Institute for Advanced Studies in Basic Sciences (IASBS), Zanjan 4513766731, Iran. Tel.: +98 2414152013.

E-mail addresses: abedinpour@iasbs.ac.ir, abedinpour@gmail.com (S.H. Abedinpour).
} 
density-density response function, and furthermore demonstrate that an undamped zero-sound mode exists for any value of the interaction strength, down to infinitesimally weak couplings.

(c) 2013 Elsevier Inc. All rights reserved.

\section{Introduction}

Recent experimental breakthroughs in trapping and cooling polar molecules and atoms with large permanent magnetic moments has triggered a considerable theoretical interest in quantum dipolar fluids [1-5]. Unlike the usual van der Waals interaction between atoms, which can be replaced by a contact Fermi pseudo-potential at ultra-low temperatures [6], the dipole-dipole interaction is long ranged and anisotropic. It is therefore natural to expect more exotic phases in ultracold dipolar gases. While one of the greatest advantages of short-range interactions is their tunability through Feshbach resonances [6,7], techniques have been proposed [8] for controlling both strength and sign of dipolar interactions as well.

As already mentioned, the inter-particle interaction between polarized dipoles (i.e. dipoles aligned in the same direction) has two important features: (i) it is long-ranged, i.e. it decays like $1 / r^{3}$ at large distances, and (ii) it is anisotropic. In particular, it is repulsive for dipoles aligned side-by-side and is attractive for dipoles aligned head-to-toe.

It is worth mentioning that the attractive part of dipole-dipole interactions can drive dipolar fluids towards instabilities. For example, in alkali-metal diatomic molecules such as $\mathrm{K}-\mathrm{Rb}$, Li-Na, etc., some chemically reactive channels, which are energetically favorable, exist and lead to particle recombination and two-body losses in the gas [5,9]. Moreover, pairing instabilities can also appear due to the attractive part of the dipole-dipole interaction.

A very simple method for stabilizing dipolar gases is to confine them into low-dimensional geometries. For example, a trap with pancake geometry together with a polarizing field, which aligns the dipoles along the direction of transverse confinement, simulates a stable two-dimensional (2D) system with purely repulsive and isotropic dipolar interactions of the form

$$
v_{\mathrm{dd}}(r)=\frac{C_{\mathrm{dd}}}{4 \pi} \frac{1}{r^{3}}
$$

Here $C_{\mathrm{dd}}$ is the dipole-dipole coupling constant, which depends on the microscopic origin of the interaction: e.g., it is $d^{2} / \epsilon_{0}$ for particles with permanent electric dipole moment $d$ and $\mu_{0} M^{2}$ for particles with permanent magnetic dipole moment $M$ (here $\epsilon_{0}$ and $\mu_{0}$ are the permittivity and permeability of vacuum, respectively).

Ground-state properties and collective modes of 2D dipolar fermions have been addressed in a number of studies [10-22]. For their particular relevance to this Article we highlight the following two recent studies [12,13] of a 2D dipolar Fermi gas with isotropic interactions as in Eq. (1). Lu and Shlyapnikov [12] have calculated a number of Fermi-liquid properties of a weakly interacting 2D dipolar Fermi gas. In particular, they have presented ground-state energy and several thermodynamic quantities up to second order in a natural dimensionless coupling constant, which we have introduced below in Eq. (3). More recently, Matveeva and Giorgini [13] have carried out quantum Monte Carlo (QMC) simulations of a 2D dipolar Fermi gas, presenting in particular results for the phase diagram of this system over a wide range of coupling constants.

In this Article we present a theoretical study of ground-state and dynamical properties of a 2D dipolar Fermi gas with average density $n$. Our main focus is on the pair distribution function $g(r)$, which is often referred to as "Pauli-Coulomb hole". This is defined so that the quantity $2 \pi n g(r) d r$ gives the average number of dipoles lying within a circular shell of radius $r$ and thickness $d r$ centered on a "reference" dipole sitting at the origin $[23,24]$. We present a self-consistent semi-analytic theory of the pair distribution function, which incorporates many-body exchange and correlation effects, thereby allowing us to explore the physics of the system at strong coupling. Our approach, which is based on the so-called Euler-Lagrange Fermi-hypernetted-chain (FHNC) approximation at 
zero temperature [25-28], involves the solution of a zero-energy scattering Schrödinger equation with a suitable effective potential [29-34]. This contains a "bosonic term" from Jastrow-Feenberg correlations and a "fermionic term" from kinetic energy and exchange, which is tailored to reproduce the Hartree-Fock (HF) limit at weak coupling and guarantees the antisymmetry of the fermionic wave function. Furthermore, we use the fluctuation-dissipation theorem [23,24] and the static structure factor obtained from the FHNC approximation to calculate the dynamical density-density linearresponse function. With this quantity at our disposal, we investigate the existence of a "zero sound" mode [23] in a 2D dipolar Fermi gas. Our results for the pair distribution function and the static structure factor have been benchmarked against state-of-the-art quantum Monte Carlo results [13].

This Article is organized as follows. In Section 2 we present our model and the self-consistent method we use to calculate in an accurate manner the pair distribution function of a 2D dipolar Fermi gas. In Section 3 we discuss a number of approximations we make to derive the dynamical density-density linear-response function of a 2D dipolar Fermi gas and explain how this can be used to examine the emergence of a collective zero-sound mode due to many-body effects. Section 4 collects our main numerical results, while Section 5 contains a brief summary of our main findings.

\section{Scattering theory for the Pauli-Coulomb hole}

We consider a spin-polarized 2D dipolar Fermi fluid described by the following first-quantized Hamiltonian [35]:

$$
\mathscr{H}=-\frac{\hbar^{2}}{2 m} \sum_{i} \nabla_{\boldsymbol{r}_{i}}^{2}+\sum_{i<j} v_{\mathrm{dd}}\left(\left|\boldsymbol{r}_{i}-\boldsymbol{r}_{j}\right|\right),
$$

where $m$ is the mass of a dipole and the bare dipole-dipole interaction has been introduced above in Eq. (1). The ground-state properties of the Hamiltonian (2) are governed by a single dimensionless parameter:

$$
\lambda=k_{\mathrm{F}} r_{0},
$$

where $r_{0}=m C_{\mathrm{dd}} /\left(4 \pi \hbar^{2}\right)$ is a characteristic length scale and $k_{\mathrm{F}}=\sqrt{4 \pi n}$ is the Fermi wave number, $n$ being the 2D average density.

In order to calculate the ground-state properties of the Hamiltonian (2), we use the FHNC [25-27] approximation at zero temperature. In what follows we first present our theory at the simplest level (which works well in the perturbative regime $\lambda \ll 1$ ) and then transcend it to obtain accurate results at strong coupling $(\lambda \gg 1)$.

With the zero of energy taken at the chemical potential, one can write a formally exact differential equation for the pair distribution function [30,31]:

$$
\left[-\frac{\hbar^{2}}{m} \nabla_{\boldsymbol{r}}^{2}+V_{\mathrm{eff}}(r)\right] \sqrt{g(r)}=0,
$$

where, as we have already introduced, $g(r)$ is defined so that the quantity $2 \pi n g(r) d r$ gives the average number of dipoles lying within a shell of radius $r$ and thickness $d r$. We write the effective scattering potential $V_{\text {eff }}(r)$ as the sum of three contributions:

$$
V_{\text {eff }}(r)=v_{\text {dd }}(r)+W_{\mathrm{B}}(r)+W_{\mathrm{F}}(r) .
$$

Here $v_{\mathrm{dd}}(r)$ is the bare repulsive dipole-dipole interaction in Eq. (1) while the bosonic contribution to the scattering potential, $W_{\mathrm{B}}(r)$, is defined, at the level of the so called "FHNC/0" approximation, by the following equation [36]:

$$
\begin{aligned}
\left.W_{\mathrm{B}}(k)\right|_{\mathrm{FHNC} / 0} & \equiv-\frac{\epsilon(k)}{2 n}[2 S(k)+1]\left[\frac{S(k)-1}{S(k)}\right]^{2} \\
& =-\frac{\epsilon(k)}{n}[S(k)-1]-V_{\mathrm{ph}}(k) .
\end{aligned}
$$


In writing Eq. (6) we have introduced the Fourier transform (FT) $W_{\mathrm{B}}(k)$ of $W_{\mathrm{B}}(r)$ according to

$$
\mathrm{FT}[F(r)] \equiv \int d^{2} \boldsymbol{r} F(r) \exp (i \boldsymbol{k} \cdot \boldsymbol{r}) .
$$

Furthermore, $\epsilon(k)=\hbar^{2} k^{2} /(2 m)$ is the single-particle energy, $S(k)$ is the instantaneous or "static" structure factor [24], $S(k)=1+n$ FT $[g(r)-1]$, and $V_{\mathrm{ph}}(k)$ is the particle-hole effective interaction [28], which is defined as

$$
S(k)=\frac{1}{\sqrt{1+2 n V_{\mathrm{ph}}(k) / \varepsilon(k)}} .
$$

When $\lambda \sim 1$ the simplest approximation for $W_{\mathrm{B}}(r)$ in Eq. (6) is inadequate. Improvements on Eq. (6) can be sought in two directions [37]. The FHNC/0 may be transcended by the inclusion of (i) low-order "elementary" diagrams and (ii) three-body Jastrow-Feenberg correlations.

The contribution from three-body correlations to the bosonic potential is given by [37]:

$$
W_{\mathrm{B}}^{(3)}(k)=\frac{1}{4 n^{2}} \int \frac{d^{2} \boldsymbol{q}}{(2 \pi)^{2}} S(p) S(q) u_{3}(\boldsymbol{q}, \boldsymbol{p}, \boldsymbol{k})\left\{v_{3}(\boldsymbol{q}, \boldsymbol{p}, \boldsymbol{k})+[E(p)+E(q)] u_{3}(\boldsymbol{q}, \boldsymbol{p}, \boldsymbol{k})\right\} .
$$

In the previous equation, $\boldsymbol{p}=-(\boldsymbol{q}+\boldsymbol{k}), E(k)=\epsilon(k) / S(k)$ is the Bijl-Feynman excitation spectrum [24],

$$
v_{3}(\boldsymbol{q}, \boldsymbol{p}, \boldsymbol{k})=\left(\hbar^{2} / m\right)[\boldsymbol{k} \cdot \boldsymbol{p} \chi(p)+\boldsymbol{k} \cdot \boldsymbol{q} \chi(q)+\boldsymbol{p} \cdot \boldsymbol{q} \chi(q)],
$$

and

$$
u_{3}(\boldsymbol{q}, \boldsymbol{p}, \boldsymbol{k})=-\frac{\left(\hbar^{2} / 2 m\right)}{E(k)+E(p)+E(q)}[\boldsymbol{k} \cdot \boldsymbol{p} \chi(k) \chi(p)+\boldsymbol{p} \cdot \boldsymbol{q} \chi(p) \chi(q)+\boldsymbol{k} \cdot \boldsymbol{q} \chi(k) \chi(q)] .
$$

In Eqs. (10)-(11) $\chi(k)=1-1 / S(k)$. We have taken into account higher-order terms that are missed by the FHNC/0 approximation by assuming that they lead to corrections to the scattering potential $V_{\text {eff }}(r)$. Using the theory developed by Apaja et al. [37], we have supplemented $\left.W_{\mathrm{B}}(k)\right|_{\mathrm{FHNC} / 0}$ in Eq. (6) by the inclusion of the three-body potential $W_{\mathrm{B}}^{(3)}(k)$ :

$$
\left.\left.W_{\mathrm{B}}(k)\right|_{\mathrm{FHNC} / \alpha 3} \equiv W_{\mathrm{B}}(k)\right|_{\mathrm{FHNC} / 0}+\alpha(\lambda) W_{\mathrm{B}}^{(3)}(k) .
$$

If $\alpha(\lambda)$ is set to unity, the right hand side of Eq. (12) defines the so-called "FHNC/3" approximation. It has been shown that the contribution of higher order correlations and elementary diagrams to the bosonic potential have a structure very similar to the three-body potential of Eq. (9) $[38,39]$. Therefore higher-order contributions beyond $\mathrm{FHNC} / 3$ can be effectively taken into account by introducing a weighting function $\alpha(\lambda)>1$ [32-34,38,39]. This approximation has been termed [34] "FHNC/ $\alpha 3$ ". A convenient analytical parametrization of the function $\alpha(\lambda)$ for 2D dipolar fluids can be found in Ref. [34]. Using the notation of this Article, it reads as follows:

$$
\alpha(\lambda)=1.88+3.26 \exp \left(-0.046 \lambda^{1.16}\right) .
$$

The previous equation is valid all the way up to the critical coupling $(\lambda \sim 25)$ for the liquid-to-crystal quantum phase transition [13].

We finally turn to describe the last term in Eq. (5), which is supposed to take care of the fermionic statistics of the problem. According to the original version of the FHNC theory [25-27], the "Fermi potential" $W_{\mathrm{F}}(r)$ has a very complicated form. Here we have decided to use a simple but effective recipe, which was first proposed by Kallio and Piilo [29] for the 3D electron liquid. In this approximate scheme $W_{\mathrm{F}}(r)$ is given by the following expression:

$$
W_{\mathrm{F}}(r)=\frac{\hbar^{2}}{m} \frac{\nabla_{\boldsymbol{r}}^{2} \sqrt{g_{\mathrm{HF}}(r)}}{\sqrt{g_{\mathrm{HF}}(r)}}-\lim _{\lambda \rightarrow 0} W_{\mathrm{B}}(r),
$$


where $g_{\mathrm{HF}}(r)$ is the well-known [23,24] 2D Hartree-Fock pair distribution function and $W_{\mathrm{B}}(r)$ is the bosonic potential defined above in Eq. (6) (at the FHNC/0 level) or in Eq. (12) (at the FHNC/ $\alpha 3$ level). The simple choice in Eq. (14) guarantees that the HF limit is recovered exactly in weak coupling $\lambda \rightarrow 0$ limit. The Fermi potential (14) has been extensively investigated for 3D [30] and 2D [32] electron liquids yielding results in excellent agreement with QMC simulation data even in the strongly correlated regime. This is simply understood as the effects of statistics is expected to weaken with increasing interaction strength.

In practice direct solution of the differential scattering Eq. (4) could be challenging. Instead one can invert it to find

$$
V_{\mathrm{ph}}(r)=g(r)\left[v_{\mathrm{dd}}(r)+W_{\mathrm{F}}(r)\right]+[g(r)-1] W_{\mathrm{B}}(r)+\frac{\hbar^{2}}{m}|\nabla \sqrt{g(r)}|^{2} .
$$

Now, Eqs. (6), (8), (14) and (15) form a closed set of equations, which can be solved numerically in a self-consistent manner: one can start from a trial guess for the static structure factor $S(k)$ to find $g(r)$ from its Fourier transform and the bosonic potential $W_{\mathrm{B}}(k)$ from Eq. (6), and then $V_{\mathrm{ph}}(r)$ from Eq. (15). The obtained value for the effective particle-hole interaction can be used to find a new value for $S(k)$ through Eq. (8). This self-consistency loop should be repeated until the desired degree of accuracy is achieved.

Once the pair distribution function has been calculated, the ground-state energy per particle of the system, $\varepsilon_{\mathrm{GS}}$, can be easily extracted by using the integration-over-the-coupling-constant algorithm [24]:

$$
\varepsilon_{\mathrm{GS}}=\varepsilon_{0}+\frac{n}{2} \int_{0}^{1} d \gamma \int d^{2} \boldsymbol{r} v_{\mathrm{dd}}(r) g_{\gamma}(r),
$$

where $\varepsilon_{0}=\varepsilon_{\mathrm{F}} / 2=\hbar^{2} k_{\mathrm{F}}^{2} /(4 m)=\hbar^{2} \lambda^{2} /\left(4 m r_{0}^{2}\right)$ is the ground-state energy of the non-interacting system, $\varepsilon_{\mathrm{F}}$ being the Fermi energy, and $g_{\gamma}(r)$ is the pair distribution function of an auxiliary system with scaled dipole-dipole interactions of the form $v_{\mathrm{dd}}^{(\gamma)}(r)=\gamma v_{\mathrm{dd}}(r)=\gamma C_{\mathrm{dd}} /\left(4 \pi r^{3}\right)$. In practice, the integration over $\gamma$ is carried out by integrating over the coupling constant $\lambda$. Note that Eq. (16) returns the exact ground-state energy of the interacting many-particle system once the exact pair distribution function is known. If instead one uses the HF pair distribution function $g_{\mathrm{HF}}(r)$, Eq. (16) gives the HF approximation for the ground-state energy.

In Section 4 we present numerical results obtained only within our most elaborate approximation, i.e. the $\mathrm{FHNC} / \alpha 3$ approximation. Nevertheless, for the sake of simplicity, all our numerical results for $g(r), S(k)$, and $\varepsilon_{\mathrm{GS}}$ will be labeled by the acronym "FHNC" (rather than "FHNC $/ \alpha 3$ ").

\section{Linear-response theory and collective modes}

Once an accurate description of the ground-state quantities like pair distribution function is obtained from e.g., FHNC or QMC, the density-density response function and dynamical structure factor can be calculated using different approximations. Correlated basis function formalism [40] is a superb example which has been successfully employed for the dynamical properties of 2D dipolar Bose gases [41,42]. Here, as we are only interested in the long wavelength behavior of the dynamical response function, we follow a much simpler approach based on the fluctuation-dissipation theorem and a static approximation for the effective inter-particle interactions, which we will explain in details below.

The density-density linear-response function of a many-particle system can be generically written as follows [24]:

$$
\chi_{n n}(k, \omega)=\frac{\chi_{0}(k, \omega)}{1-\psi(k, \omega) \chi_{0}(k, \omega)} \equiv \frac{\chi_{0}(k, \omega)}{\varepsilon(k, \omega)},
$$

where $\psi(k, \omega)$ is a suitable dynamical effective potential - not to be confused with the Fourier transform of the effective potential $V_{\text {eff }}(r)$ which enters the zero-energy scattering Schrödinger 
equation $(4)$ - and $\chi_{0}(k, \omega)$ is the well-known [24,43] density-density response function of an ideal (i.e. non-interacting) 2D Fermi gas.

In the celebrated Random Phase Approximation (RPA) [23,24], the effective potential $\psi(k, \omega)$ is brutally approximated with the FT of the bare inter-particle potential, i.e. $v_{\mathrm{dd}}(r)$ in our case. It is very well known $[23,24]$ that the RPA neglects short-range exchange and correlation effects and that it is intrinsically a weak-coupling theory. It is thus not expected to work well (in reduced spatial dimensions and) for values of the dimensionless coupling constant $\lambda \gtrsim 1$. One of the main drawbacks of the RPA is that it grossly overestimates the strength of the Pauli-Coulomb hole by predicting large and negative values for $g(r)$ at short distances, thereby violating the fundamental requirement of $g(r)>0$. Moreover, in the context of dipolar Fermi gases, the RPA predicts that the long-wavelength collective excitation spectrum (zero-sound mode) is empathic to the short-range details of the bare interaction potential [14], i.e. the ultraviolet cut-off which is needed to regularize the Fourier transform of the bare dipole-dipole potential $v_{\mathrm{dd}}(r)$. Another problem with RPA for dipolar Fermi fluids is that it fails to correctly describe even the long wavelength regime [17].

In the past sixty years or so, a wide body of literature has been devoted to transcend the RPA, especially in the context of 2D electron liquids in semiconductor heterojunctions [24]. Following the seminal works by Hubbard [44] and Singwi, Tosi, Land, and Sjölander [45] (STLS), one successful route has been based on the use of "local-field factors" [24,46]. Here we will not use Hubbard or STLS local-field factors. (For a successful employment of the STLS approximation in the context of 2D dipolar Fermi gases see, for example, Ref. [17].) In this Article we would like to construct a reliable approximation for the density-density response function $\chi_{n n}(k, \omega)$, which is based on the FHNC theory of the pair distribution function outlined in Section 2.

We thus start from the well-known fluctuation-dissipation theorem [24], which relates the imaginary part of density-density response function $\chi_{n n}(k, \omega)$ to the instantaneous structure factor $S(k)$. At zero temperature this theorem reads [24]

$$
S(k)=-\frac{\hbar}{\pi n} \int_{0}^{\infty} d \omega \Im m\left[\chi_{n n}(k, \omega)\right] .
$$

To make some progress, we neglect the frequency dependence of the effective potential $\psi(k, \omega)$ in Eq. (17): we replace the complex function $\psi(k, \omega)$ by a real quantity, which we denote by the symbol $\bar{\psi}(k)$. This approximation is often made in treating correlation effects in the electron liquid [23,24] and is certainly shared by the most elementary theories based on local-field factors (Hubbard and STLS). In this case, one can view Eq. (18) as an integral equation for the unknown quantity $\bar{\psi}(k)$, assuming that the left hand side of Eq. (18), i.e. the static structure factor, is accurately known, e.g. from QMC simulations or microscopic theories such as the one outlined in Section 2. This fully numerical approach has been successfully employed in different contexts $[47,48]$. The physical interpretation of $\bar{\psi}(k)$ is clear: it represents the "best" average effective potential (averaged over frequency, as from Eq. (18)) which, by virtue of the fluctuation-dissipation theorem, makes the response of the system consistent with the local structure of the fluid around a reference dipole (the Pauli-Coulomb hole).

In the spirit of making the problem at hand more amenable to a semi-analytical treatment, we also use the so-called "mean-spherical approximation" for $\chi_{0}(k, \omega)[48]$ :

$$
\chi_{0}^{(\mathrm{MSA})}(k, \omega) \equiv \frac{2 n \epsilon(k)}{\left(\hbar \omega+i 0^{+}\right)^{2}-\left[\epsilon(k) / S_{\mathrm{HF}}(k)\right]^{2}},
$$

where $S_{\mathrm{HF}}(k)$ is the $2 \mathrm{D}$ HF static structure factor [24]. This approximation allows us to perform the integration over $\omega$ in Eq. (18) analytically, yielding

$$
\bar{\psi}(k) \stackrel{\mathrm{MSA}}{=} \frac{\epsilon(k)}{2 n}\left[\frac{1}{S^{2}(k)}-\frac{1}{S_{\mathrm{HF}}^{2}(k)}\right] .
$$

For the static structure factor $S(k)$ on the right hand side of Eq. (20) we use the FHNC theory described above in Section 2.

We can now use Eqs. (17) and (20) to study the existence of a collective mode [24] in the density channel (zero sound [23]). This is the solution of the complex equation $\varepsilon(k, \omega)=0$ or, equivalently, 
of the following two real equations:

$$
\left\{\begin{array}{l}
1-\bar{\psi}(k) \Re e\left[\chi_{0}(k, \omega)\right]=0 \\
\Im m\left[\chi_{0}(k, \omega)\right]=0 .
\end{array}\right.
$$

The solution $\omega_{\mathrm{ZS}}=\omega_{\mathrm{ZS}}(k)$ of Eq. (21) corresponds to a self-sustained oscillation with a non-trivial dispersion relation and a finite velocity $v_{\mathrm{ZS}} \equiv \lim _{k \rightarrow 0} \omega_{\mathrm{ZS}}(k) / k$ in the long-wavelength limit. The second equation means that the collective mode is undamped when it falls in the region of $(k, \omega)$ space where particle-hole pairs are absent. This occurs when $v_{\mathrm{ZS}}>v_{\mathrm{F}}, v_{\mathrm{F}}=\hbar k_{\mathrm{F}} / m$ being the Fermi velocity. When the collective mode enters the particle-hole continuum, Landau damping starts: the mode has sufficient energy to decay by emitting a particle-hole pair while, at the same time, conserving momentum.

Before concluding this section, we derive a formal expression for the zero sound velocity, $v_{\mathrm{ZS}}$, in terms of $\bar{\psi}(0)$. (As we will see below in Section $4, \bar{\psi}(k)$ is regular and positive at $k=0$.) In order to find $v_{\mathrm{ZS}}$ we use the following long-wavelength limit of the ideal response function $[49,24]$ :

$$
\lim _{k \rightarrow 0} \Re e\left[\chi_{0}\left(k, v_{\mathrm{F}} k v\right)\right]=-N(0)\left(1-\frac{v}{\sqrt{v^{2}-1}}\right),
$$

where $N(0)=m /\left(2 \pi \hbar^{2}\right)$ is the 2D density-of-states at the Fermi energy and $v=\omega /\left(v_{\mathrm{F}} k\right)=$ constant. Note that, in Eq. (22), the ratio between $\omega=v_{\mathrm{F}} k v$ and $k$ remains constant in the limit $k \rightarrow 0$, precisely as in the zero sound mode [ $\left[\lim _{k \rightarrow 0} \omega_{\mathrm{Zs}}(k) / k=\right.$ constant]. It is very important to observe that the asymptotic behavior (22) needed for the calculation of the zero sound velocity is very different from the usual high-frequency limit imposed by the $f$-sum rule [24]:

$$
\Re e\left[\chi_{0}\left(k, \omega \gg v_{\mathrm{F}} k\right)\right]=\frac{n k^{2}}{m \omega^{2}} .
$$

Now, replacing Eq. (22) (and not Eq. (23)) in Eq. (21), we find the following formal expression for the zero sound velocity in units of the Fermi velocity:

$$
\frac{v_{\mathrm{ZS}}}{v_{\mathrm{F}}}=\frac{1+N(0) \bar{\psi}(0)}{\sqrt{1+2 N(0) \bar{\psi}(0)}},
$$

which is well defined if $\bar{\psi}(0)>-[2 N(0)]^{-1}$. Note that the quantity on the right hand side of Eq. (24) is always larger than one. We therefore conclude that, within the approximations we made to derive Eq. (20), a 2D dipolar Fermi gas always displays (i.e. for every value of the coupling constant $\lambda$ ) an undamped zero sound mode, in agreement with Ref. [12]. As discussed at length in Ref. [12], this mode stems entirely from correlation effects and it is thus not describable within the HF approximation. However, even the RPA is not enough in this respect since it yields a zero sound mode with a velocity that depends on the short-range cut off of the bare dipole-dipole interaction [14]. A serious theory of the zero sound mode thus requires the inclusion of correlation effects. The FHNC theory discussed in this Article is an example.

\section{Numerical results and discussion}

In this section we present our main numerical results.

We begin by showing our results for the pair distribution function $g(r)$ and static structure factor $S(k)$. In Figs. 1 and 2 we compare our results (lines) with the corresponding QMC data (symbols) [13]. The agreement between theory and numerical simulations is clearly excellent up to very large values of the dimensionless coupling constant $\lambda(\lambda=20)$. At these values of $\lambda$, conventional theories such as RPA and STLS fail, even qualitatively. For example, both RPA and STLS seriously underestimate at strong coupling the amplitude of the first-neighbor peak and, even more dramatically, yield on-top values of the pair distribution function, $g(0)$, which are negative, thereby violating the fundamental requirement $g(r)>0 \forall r$. Note that, according to the QMC study by Matveeva and Giorgini [13], a 


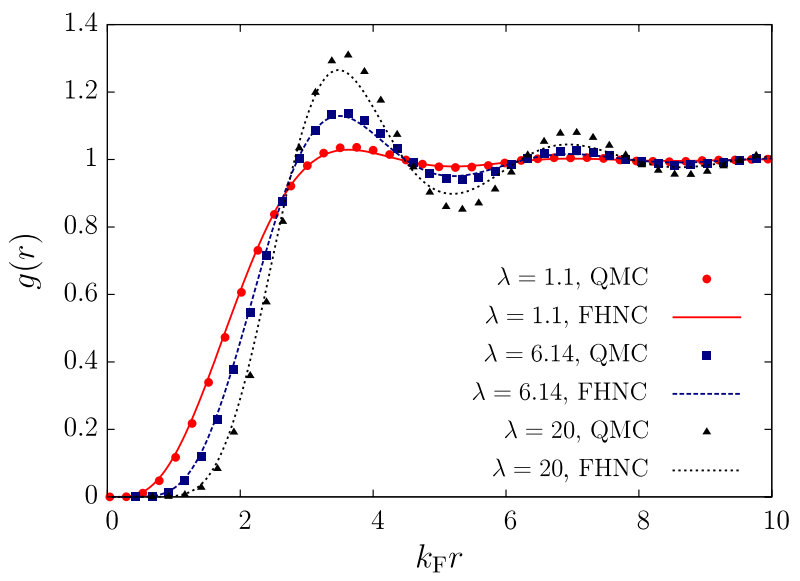

Fig. 1. (Color online) The pair distribution function $g(r)$ of a 2D fluid of dipolar fermions is plotted as a function of the scaled distance $k_{\mathrm{F}} r$ and for various values of the dimensionless coupling constant $\lambda$. In this plot, lines label the results of the FHNC approximation (this work) while symbols label QMC results [13].

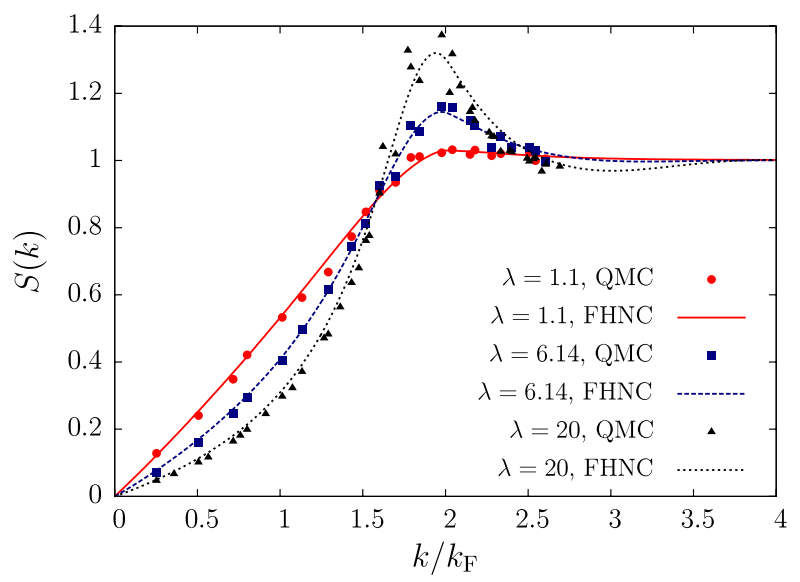

Fig. 2. (Color online) The instantaneous structure factor $S(k)$ of a $2 \mathrm{D}$ fluid of dipolar fermions is plotted as a function of $k / k_{\mathrm{F}}$ and for various values of $\lambda$. In this plot, lines label the results of the FHNC approximation (this work) while symbols label QMC results [13].

liquid-to-crystal quantum phase transition is expected to occur at $\lambda \sim 25$. This is clearly signaled by the amplitude of the first-neighbor peak in the static structure factor (see Fig. 2), which increases with increasing $\lambda$ indicating the build up of correlations in the liquid phase upon approaching crystalline order.

The pair distribution function shown in Fig. 1 can be used to calculate the ground-state energy by employing Eq. (16). Since our FHNC results for the pair distribution function are in very good agreement with the QMC data, which are typically believed to be nearly exact [50], we expect the corresponding FHNC values for the ground-state energy to be very close to QMC fluid-state energy. In Fig. 3 we report our results for the ground-state energy as obtained from the pair distribution function calculated at the FHNC level. In the same plot we compare our findings with the corresponding QMC results [13] of the homogeneous fluid phase. Note that beyond the liquid to crystal quantum phase transition coupling strength, the crystal phase has a lower ground-state energy than the homogeneous fluid phase. One can use the homogeneous solution of the EL-FHNC equations as an input for the density-functional theory to find the quantum phase transition [51]. In passing, we note that our FHNC 


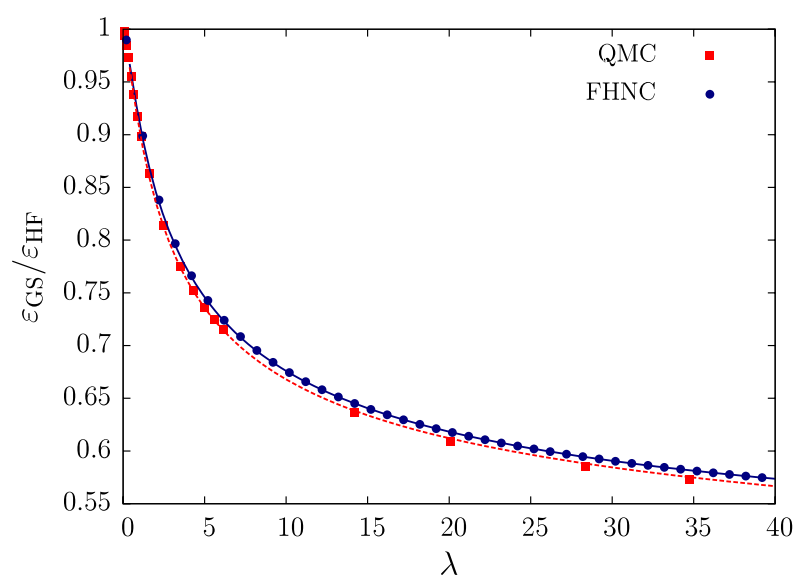

Fig. 3. (Color online) The ground-state energy of a 2D fluid of dipolar fermions (in units of the Hartree-Fock energy $\varepsilon_{\mathrm{HF}} / \varepsilon_{0}=$ $1+128 \lambda /(45 \pi)$ ), is plotted as a function of $\lambda$. Blue circles label the results of the FHNC approximation (this work) while red squares label the QMC results [13]. The solid line represents the parametrization formula in Eq. (25) with $a=1.5006$, $b=1.0107$, and $c=0$. The dashed line represents the parametrization in Eq. (25) with $a=\sqrt{\zeta_{3}}$ (see main text), $b=1.1017$, and $c=-0.0100$.

results for the ground-state energy (per particle) can be accurately parametrized by the following expression:

$$
\varepsilon_{\mathrm{GS}}(\lambda)=\varepsilon_{0}\left[1+\frac{128}{45 \pi} \lambda-\frac{\lambda^{2}}{2} \ln \left(1+\frac{1}{a \sqrt{\lambda}+b \lambda+c \lambda^{3 / 2}}\right)\right],
$$

where $a, b$, and $c$ are numerical constants. The sum of the first two terms in square brackets on the right hand side of Eq. (25) yields the HF approximation for the ground-state energy [12]: $\varepsilon_{\mathrm{HF}} \equiv$ $\varepsilon_{0}[1+128 \lambda /(45 \pi)]$. The best fit of our FHNC data for the energy of the liquid phase up to $\lambda=40$ is obtained by using $a$ and $b$ as free fitting parameters and setting $c=0$ : we find $a=1.5006$ and $b=1.0107$. The result of this two-parameter fit is shown in Fig. 3 (solid line).

Alternatively, the simple formula in Eq. (25) can be used to parametrize also the QMC data by Matveeva and Giorgini [13]. Since these data are believed to be very close to the exact values, we can fix the value of $a$ by imposing that Eq. (25) reproduces exactly the results of second-order perturbation theory [12]. Straightforward algebraic manipulations on Eq. (25) yield the following expansion in powers of $\lambda$ for $\lambda \rightarrow 0$ :

$$
\varepsilon_{\mathrm{GS}}(\lambda)=\varepsilon_{0}\left[1+\frac{128}{45 \pi} \lambda+\frac{\lambda^{2}}{4} \ln \left(a^{2} \lambda\right)+\cdots\right],
$$

where "..." denotes higher-order terms. To the same order of perturbation theory, Lu and Shlyapnikov [12] find (Eq. (91) in their work):

$$
\varepsilon_{\mathrm{GS}}(\lambda)=\varepsilon_{0}\left[1+\frac{128}{45 \pi} \lambda+\frac{\lambda^{2}}{4} \ln \left(\zeta_{3} \lambda\right)+\cdots\right]
$$

where $\zeta_{3}=1.43$ (we have taken the limit $A \rightarrow 0$ in the expression for $\zeta_{3}$ given in Ref. [12]). Comparing Eq. (26) with Eq. (27) we conclude that $a=\sqrt{\zeta_{3}} \sim 1$.2. The parameters $b$ and $c$ can then be used to yield the best fit to the QMC data for the energy of the liquid phase up to $\lambda=72$ [13]: we find $b=1.1017$ and $c=-0.0100$. The result of this two-parameter fit is also shown in Fig. 3 (dashed line).

The difference between the total ground-state energy and the non-interacting contribution $\varepsilon_{0}$ defines the interaction energy: $\varepsilon_{\text {int }}(\lambda)=\varepsilon_{\mathrm{GS}}(\lambda)-\varepsilon_{0}(\lambda)$. Note that unlike the jellium model for electron gases [24], the Hartree contribution to the interaction energy does not vanish in our system of 


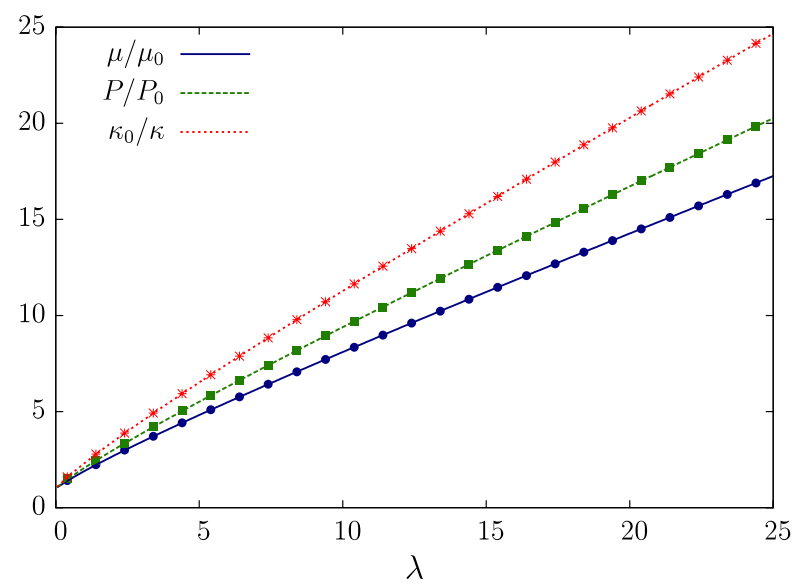

Fig. 4. (Color online) Three important thermodynamic quantities: the chemical potential $\mu$, pressure $P$, and inverse compressibility $\kappa^{-1}$ of a 2D fluid of dipolar fermions (in units of their non-interacting values) are plotted as functions of $\lambda$. Lines label analytic results obtained from the parametrization formula (25) while symbols label numerical results obtained directly from the FHNC ground-state energy.

polarized dipolar Fermi gases [12]. Eq. (25) thus provides an extremely useful input for calculations of ground-state properties of inhomogeneous 2D dipolar Fermi gases based on density functional theory (DFT) [24]. In DFT, indeed, one needs to approximate the unknown interaction energy $E_{\text {int }}[n(\boldsymbol{r})]$, viewed as a functional of the local ground-state density $n(\boldsymbol{r})$. In the local density approximation (LDA) one can write [24]

$$
E_{\text {int }}[n(\boldsymbol{r})] \stackrel{\text { LDA }}{=} \int d^{2} \boldsymbol{r} n(\boldsymbol{r}) \varepsilon_{\text {int }}(\lambda(\boldsymbol{r})),
$$

where $\lambda(\boldsymbol{r})$ is defined as in Eq. (3) with $n$ replaced by the local density $n(\boldsymbol{r})$. An example where the DFT-LDA approach could be very useful is a 2D dipolar Fermi gas in the presence of an in-plane harmonic confinement potential $V_{\text {ext }}=\sum_{i} m \omega^{2} \boldsymbol{r}_{i}^{2} / 2$.

From the knowledge of the ground-state energy (per particle) $\varepsilon_{\mathrm{GS}}$ we can also construct a number of thermodynamic quantities at zero temperature. Most notably, the chemical potential $\mu=$ $\partial\left(n \varepsilon_{G S}\right) / \partial n$, the pressure $P=n^{2} \partial \varepsilon_{G S} / \partial n$, and the inverse compressibility $\kappa^{-1}=n \partial P / \partial n$ are readily obtained from the interpolation formula given in Eq. (25). We display these quantities as functions of the interaction strength $\lambda$ in Fig. 4 . Note that all these quantities, which still remain to be experimentally measured, are strongly enhanced by interactions.

Fig. 5 illustrates the effective potential $\bar{\psi}(k)$ as obtained from Eq. (20). We clearly see from this plot that $\bar{\psi}(k)$ is regular and positive at $k=0$.

Finally, in Fig. 6 we illustrate our predictions for the dispersion of the zero sound mode. As already discussed at the end of Section 3, our theory predicts an undamped zero sound mode at long wavelengths for every value of $\lambda$. The zero sound velocity as well as the critical wave vector at which Landau damping starts to increase with increasing $\lambda$.

\section{Summary}

In summary, we have presented an extensive study of ground-state and dynamical properties of a strongly correlated two-dimensional spin-polarized fluid of dipolar fermions.

The main focus of our work has been on the pair distribution function $g(r)$, a key groundstate property of any quantum fluid. To calculate the pair distribution function we have employed the Fermi-hypernetted-chain approximation combined with a zero-energy scattering Schrödinger equation for the "pair amplitude" $\sqrt{g(r)}$. The effective potential that enters this equation includes a bosonic term from Jastrow-Feenberg correlations and a fermionic contribution from kinetic energy 


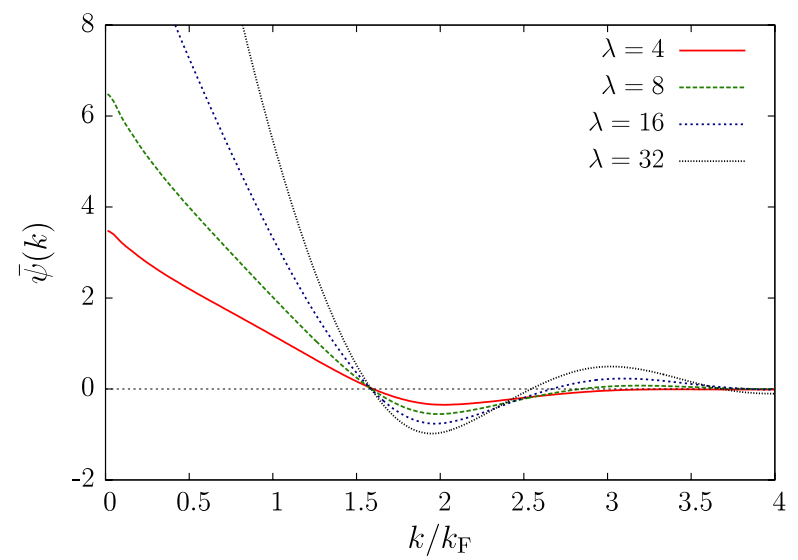

Fig. 5. (Color online) The effective interaction $\bar{\psi}(k)$ (in units of $2 \pi \hbar^{2} / \mathrm{m}$ ) in a 2D fluid of dipolar fermions as obtained from Eq. (20) is plotted as a function of $k / k_{\mathrm{F}}$ for various values of $\lambda$. Note that $\bar{\psi}(k \rightarrow 0)$ tends to a positive value.

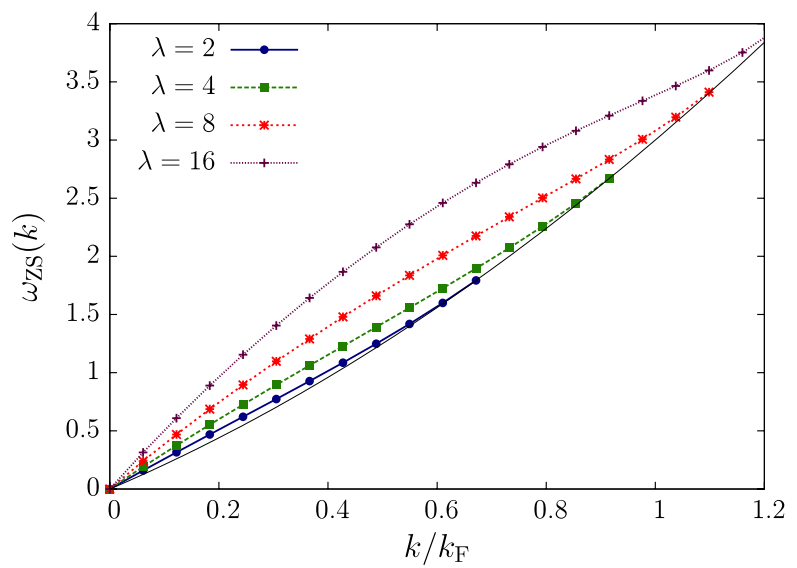

Fig. 6. (Color online) The frequency $\omega_{\mathrm{ZS}}(k)$ of the zero-sound mode in a 2D fluid of dipolar fermions is plotted as a function of $k / k_{\mathrm{F}}$ for various values of $\lambda$. The thin (black) solid line represents the upper bound of the particle-hole continuum, i.e. $\omega_{+}(k)=\epsilon(k) / \hbar+v_{\mathrm{F}} k$. Note that the zero-sound mode lies above the particle-hole continuum for every $\lambda$, up to a $\lambda$-dependent critical wave vector at which Landau damping starts.

and exchange, which is tailored to reproduce the Hartree-Fock limit at weak coupling. Our results for the pair distribution function and the static structure factor $S(k)$ have been severely benchmarked against state-of-the-art quantum Monte Carlo results by Matveeva and Giorgini [13]. Very good agreement with these results has been achieved over a wide range of coupling constants up to the liquid-to-crystal quantum phase transition.

By combining our knowledge on the pair distribution function with the fluctuation-dissipation theorem, we have been able to calculate in an approximate fashion also the dynamical density-density response function. This ingredient has been used to demonstrate that, in a two-dimensional spinpolarized fluid of dipolar fermions, an undamped zero-sound mode exists for any value of the interaction strength, down to infinitesimally weak couplings (in agreement with Ref. [12]).

Last but not least, we have presented a useful parametrization formula, Eq. (25), for the groundstate energy of a two-dimensional spin-polarized fluid of dipolar fermions, which fits well both our Fermi-hypernetted-chain results and the quantum Monte Carlo data by Matveeva and Giorgini [13]. This can be very effectively employed in density-functional calculations of 2D inhomogeneous dipolar fermions. 


\section{Acknowledgments}

We are indebted to Natalia Matveeva and Stefano Giorgini for providing us with their QMC data. It is also a pleasure to thank Nikolaj Zinner for useful discussions. B.T. acknowledges support from TUBITAK (through grants 109T267, 112T176, 210T050) and TUBA.

\section{References}

[1] M.A. Baranov, Phys. Rep. 464 (2008) 71.

[2] T. Lahaye, C. Menotti, L. Santos, M. Lewenstein, T. Pfau, Rep. Progr. Phys. 72 (2009) 126401.

[3] K. Aikawa, A. Frisch, M. Mark, S. Baier, A. Rietzler, R. Grimm, F. Ferlaino, Phys. Rev. Lett. 108 (2012) 210401.

[4] M. Lu, N.Q. Burdick, B.L. Lev, Phys. Rev. Lett. 108 (2012) 215301.

[5] M.A. Baranov, M. Dalmonte, G. Pupillo, P. Zoller, Chem. Rev. 112 (2012) 5012.

[6] C.J. Pethick, H. Smith, Bose-Einstein Condensation in Dilute Gases, Cambridge University Press, 2008; I. Bloch, J. Dalibard, W. Zwerger, Rev. Modern Phys. 80 (2008) 885;

S. Giorgini, L.P. Pitaevskii, S. Stringari, Rev. Modern Phys. 80 (2008) 1215.

[7] C. Chin, R. Grimm, Rev. Modern Phys. 82 (2010) 1225.

[8] S. Giovanazzi, A. Görlitz, T. Pfau, Phys. Rev. Lett. 89 (2002) 130401.

[9] P.S. Zuchowski, J.M. Hutson, Phys. Rev. A 81 (2010) 060703(R).

[10] G.M. Bruun, E. Taylor, Phys. Rev. Lett. 101 (2008) 245301; 107 (2011) 169901(E).

[11] C.-K. Chan, C. Wu, W.-C. Lee, S. Das Sarma, Phys. Rev. A 81 (2010) 023602.

[12] Z.K. Lu, G.V. Shlyapnikov, Phys. Rev. A 85 (2012) 023614.

[13] N. Matveeva, S. Giorgini, Phys. Rev. Lett. 109 (2012) 200401.

[14] Q. Li, E.H. Hwang, S. Das Sarma, Phys. Rev. B 82 (2010) 235126.

[15] L.M. Sieberer, M.A. Baranov, Phys. Rev. A 84 (2011) 063633.

[16] M. Babadi, E. Demler, Phys. Rev. A 84 (2011) 033636.

[17] M.M. Parish, F.M. Marchetti, Phys. Rev. Lett. 108 (2012) 145304.

[18] F.M. Marchetti, M.M. Parish, Phys. Rev. B 87 (2013) 045110.

[19] Y. Yamaguchi, T. Sogo, T. Ito, T. Miyakawa, Phys. Rev. A 82 (2010) 013643.

[20] K. Sun, C. Wu, S. Das Sarma, Phys. Rev. B 82 (2010) 075105.

[21] M. Babadi, E. Demler, Phys. Rev. B 84 (2011) 235124.

[22] N. Zinner, G.M. Bruun, Eur. Phys. J. D 65 (2011) 133.

[23] D. Pines, P. Noziéres, The Theory of Quantum Liquids, W.A. Benjamin, Inc, New York, 1966.

[24] G.F. Giuliani, G. Vignale, Quantum Theory of the Electron Liquid, Cambridge University Press, Cambridge, 2005.

[25] L.J. Lantto, P.J. Siemens, Nucl. Phys. A 317 (1979) 55; L.J. Lantto, Phys. Rev. B 22 (1980) 1380; 36 (1987) 5160.

[26] J.G. Zabolitzky, Phys. Rev. B 22 (1980) 2353.

[27] E. Krotscheck, M. Saarela, Phys. Rep. 232 (1993) 1.

[28] A. Polls, F. Mazzanti, in: A. Fabrocini, S. Fantoni, E. Krotscheck (Eds.), Introduction to Modern Methods of Quantum ManyBody Theory and Their Applications, World Scientific Publishing Company, 2002.

[29] A. Kallio, J. Piilo, Phys. Rev. Lett. 77 (1996) 4237.

[30] B. Davoudi, R. Asgari, M. Polini, M.P. Tosi, Phys. Rev. B 68 (2003) 155112.

[31] B. Davoudi, R. Asgari, M. Polini, M.P. Tosi, Phys. Rev. B 67 (2003) 172503.

[32] R. Asgari, B. Davoudi, M.P. Tosi, Solid State Commun. 131 (2004) 301.

[33] S.H. Abedinpour, R. Asgari, M. Polini, M.P. Tosi, Solid State Commun. 144 (2007) 65

[34] S.H. Abedinpour, R. Asgari, M. Polini, Phys. Rev. A 86 (2012) 043601.

[35] See, for example G.E. Astrakharchik, J. Boronat, I.L. Kurbakov, Yu.E. Lozovik, Phys. Rev. Lett. 98 (2007) 060405.

[36] T. Chakraborty, Phys. Rev. B 25 (1982) 3177; 26 (1982) 6131; T. Chakraborty, A. Kallio, L.J. Lantto, P. Pietiläinen, Phys. Rev. B 27 (1983) 3061.

[37] V. Apaja, J. Halinen, V. Halonen, E. Krotscheck, M. Saarela, Phys. Rev. B 55 (1997) 12925; R.A. Smith, A. Kallio, M. Puoskari, P. Toropainen, Nucl. Phys. A 328 (1979) 186.

[38] Q.N. Usmani, B. Friedman, V.R. Pandharipande, Phys. Rev. B 25 (1982) 4502.

[39] E. Krotscheck, Phys. Rev. B 33 (1986) 3158.

[40] E. Krotscheck, in: A. Fabrocini, S. Fantoni, E. Krotscheck (Eds.), Introduction to Modern Methods of Quantum Many-Body Theory and Their Applications, World Scientific Publishing Company, 2002.

[41] F. Mazzanti, R.E. Zillich, G.E. Astrakharchik, J. Boronat, Phys. Rev. Lett. 102 (2009) 110405.

[42] A. Macia, D. Hufnagl, F. Mazzanti, J. Boronat, R.E. Zillich, Phys. Rev. Lett. 109 (2012) 235307.

[43] F. Stern, Phys. Rev. Lett. 18 (1967) 546.

[44] J. Hubbard, Proc. R. Soc. A 243 (1957) 336.

[45] K.S. Singwi, M.P. Tosi, R.H. Land, A. Sjölander, Phys. Rev. 176 (1968) 589.

[46] For reviews see K.S. Singwi, M.P. Tosi, Solid State Phys. 36 (1981) 177; S. Ichimaru, Rev. Modern Phys. 54 (1982) 1017.

[47] J. Boronat, J. Casulleras, V. Grau, E. Krotscheck, J. Springer, Phys. Rev. Lett. 91 (2003) 085302.

[48] R. Asgari, A.L. Subaşı, A.A. Sabouri-Dodaran, B. Tanatar, Phys. Rev. B 74 (2006) 155319.

[49] G.E. Santoro, G.F. Giuliani, Phys. Rev. B 37 (1988) 937.

[50] See, for example D.M. Ceperley, in: G.F. Giuliani, G. Vignale (Eds.), The Electron Liquid Paradigm in Condensed Matter Physics, IOS Press, Amsterdam, 2005.

[51] N. Choudhury, S.K. Ghosh, Phys. Rev. B 51 (1995) 2588. 